\title{
Recognition Analysis and Simulation Implementation Based on High-Order Cumulants of Wireless Digital Modulation Mode
}

\author{
Luyong Ren, Yuanchang Wang, Qian Xi \\ College of Computer Science and Technology, Shandong University of Technology, Zibo, China \\ Email: renly@sdut.edu.cn
}

How to cite this paper: Ren, L.Y., Wang, Y.C. and Xi, Q. (2021) Recognition Analysis and Simulation Implementation Based on High-Order Cumulants of Wireless Digital Modulation Mode. Journal of Computer and Communications, 9, 15-26. https://doi.org/10.4236/jcc.2021.910002

Received: August 25, 2021

Accepted: September 27, 2021

Published: September 30, 2021

Copyright (c) 2021 by author(s) and Scientific Research Publishing Inc. This work is licensed under the Creative Commons Attribution International License (CC BY 4.0).

http://creativecommons.org/licenses/by/4.0/

(c) (i) Open Access

\begin{abstract}
This paper mainly studies the data characteristics of high order cumulants using digitally modulated signals, and constructs the identification feature parameters that can distinguish the signal modulation type by the high-order cumulants data of the digital modulation signal. Set the identification signal modulation type determination threshold based on the value of the identification feature parameter. The identification feature parameter value of the signal modulation type is compared with the set determination threshold, to realize the recognition of digital modulation signal. This identification method is implemented based on MATLAB design, with a 2ASK (2-ary Amplitude Shift Keying) signal, 4ASK (4-ary Amplitude Shift Keying) signal, 2PSK (2-ary Phase Shift Keying) signal, 4PSK (4-ary Phase Shift Keying) signal, 2FSK (2-ary Frequency Shift Keying) signal, 4FSK (4-ary Frequency Shift Keying) signal. The second, fourth and sixth order cumulants of the six signals were analyzed. Calculate the selected identification feature parameter value and the determination threshold to identify the six signals. The six signals have made MATLAB identification simulation. Simulation results show that this method is feasible and has high recognition rate. Simulation results verify that such recognition methods maintain a high recognition rate under conditions with low signal-to-noise ratio. This identification method can be extended to more MASK (M-ary Amplitude Shift Keying), MPSK (M-ary Phase Shift Keying), MFSK (M-ary Frequency Shift Keying), MQAM (M-ary Quadrature Amplitude Modulation) signal identification.
\end{abstract}

\section{Keywords}

Modulation Recognition, High-Order Cumulants, Recognition Rate, 
Recognition Methods

\section{Introduction}

Today, with the rapid development of modern digital communication technology, modulation recognition of digital signals is a key technology in communication. Modulation recognition technology has great application value in civil field, in order to ensure the normal communication of the legal user. It is necessary for the management department to identify and control the transmission signal. In the non-civil field, the modulation recognition technology of digital communication signals is applied more widely, such as electronic countermeasure, ECM, threat detection and so on [1]. At present, with the increasing variety and complexity of digital communication systems, the application environment of communication signals is becoming more and more complex, and how to recognize useful communication signals and improve the modulation recognition rate of communication signals in complex interference channels is still an important problem to be studied. In practical wireless communication, the recognition rate of digital signal modulation is affected by many factors, such as too small intensity of received signal, complex modulation mode of digital signal, complex noise interference and so on. This makes the modulation recognition of digital signals more difficult. Because of the importance of its practical application, the research of digital communication signal modulation in the complex receiving environment is very important and valuable in both academic and engineering fields. The defect of digital signal recognition based on high-order cumulant is that it has a large amount of computation. How to correctly identify the modulation signal with as little computation as possible by selecting appropriate characteristic parameters is a problem to solve. Based on the characteristic parameters of higher-order cumulants, this paper completes the modulation recognition of digital signals, and uses MATLAB to establish the software recognition simulation system of this recognition method. With the help of MATLAB simulation design and analysis, the feasibility of the identification method in this paper is verified. The pattern recognition of digital signal modulation is realized by programming in this simulation platform, which can be applied to the research and product development of signal modulation recognition technology in software radio system. This not only reduces the development cost but also shortens the development time, which provides the reference for the future communication system product technology research and development.

\section{Analysis of Digital Signal Cumulants}

The high-order cumulant of the digital signal reflects its high-order statistical characteristics, and the high-order statistics have very good anti-fading ability. Therefore, when identifying the modulation mode of a digital communication 
signal in a weak channel environment, a modulation recognition method that calculates high-order statistics of the signal can be used [2].

\section{Calculation of the Theoretical Value of the High-Order Cumulant of the Digital Signal}

In practical applications, assuming that the noise is a random process with various states, the carrier frequency of the received modulated signal has been estimated by the zero-crossing detection method. The noise-containing digital modulation signal is output through a filter at the receiving end, and then subjected to mixing processing, and the signal expression to be identified can be obtained (1) [3] [4] [5] [6],

$$
e_{k}=x_{k}+n_{k}=\sqrt{p} \mathrm{e}^{j \phi_{c}} a_{k}+n_{k}
$$

In Formula (1), $k=1,2,3, \cdots, N, \phi_{c}$ is the initial phase, $p$ is the energy of the transmitted symbol, $a_{k}$ is the transmitted symbol sequence, $n_{k}$ is the Gaussian white noise sequence.

According to the properties of the higher-order cumulants, the cumulants of the Additive white Gaussian noise whose order is larger than the second-order cumulants are always zero. Since the digital communication signals are modulated in different ways and their cumulants are different, the cumulants of different modulation signals are calculated and analyzed separately.

The procedure for calculating the cumulants of each order of the modulated digital signal is as follows: the expressions of the Mask Signal, the MFSK signal and the MPSK signal without considering the noise interference are shown in the Formula (2), in which the $a_{k}$ code element sequence values are different for different modulation modes, the second, fourth and sixth order higher moments of the corresponding modulation signal are calculated according to the $a_{k}$ code sequence. The higher-order moments are replaced by the moments and cumulants, and the theoretical values of the cumulants corresponding to the modulation modes are obtained.

$$
x_{k}=\sqrt{p} \mathrm{e}^{j \phi_{c}} a_{k}
$$

1) 2ASK signal 2nd, 4th, 6th order cumulant calculation

Derive the expressions (3) - (8) to calculate the 2nd, 4th, and 6th moments of the 2ASK signal.

$$
\begin{gathered}
M_{20}=E[x(k), x(k)]=E\left[p \mathrm{e}^{j \phi_{c}} a_{k}^{2}\right]=p \mathrm{e}^{j \phi_{c}} \\
M_{21}=E\left[x(k), x^{*}(k)\right]=E\left[p a_{k}^{2}\right]=p \\
M_{40}=E[x(k), x(k), x(k), x(k)]=E\left[p^{2} \mathrm{e}^{j 4 \phi_{c}} a_{k}^{4}\right]=2 p^{2} \mathrm{e}^{j 4 \phi_{c}} \\
M_{41}=E\left[x(k), x(k), x(k), x^{*}(k)\right]=E\left[p^{2} \mathrm{e}^{j 2 \phi_{c}} a_{k}^{4}\right]=2 p^{2} \mathrm{e}^{j 2 \phi_{c}} \\
M_{42}=E\left[x(k), x(k), x^{*}(k), x^{*}(k)\right]=E\left[p^{2} a_{k}^{4}\right]=2 p^{2} \\
M_{60}=E[x(k), x(k), x(k), x(k), x(k), x(k)]=E\left[p^{3} \mathrm{e}^{j 6 \phi_{c}} a_{k}^{6}\right]=4 p^{3} \mathrm{e}^{j 6 \phi_{c}}
\end{gathered}
$$


Substituting Formulas (3) - (8) into the moment-cumulant conversion formula, the expressions (9) - (14) for calculating the theoretical value of the 2 nd, 4 th, and 6 th order cumulants of the 2 ASK signal are obtained.

$$
\begin{gathered}
C_{20}=M_{20}=p \mathrm{e}^{j 2 \phi_{c}} \\
C_{21}=M_{21}=p \\
C_{40}=M_{40}-3 M_{20}^{2}=-p^{2} \mathrm{e}^{j 4 \phi_{c}} \\
C_{41}=M_{41}-3 M_{20} M_{21}=-p^{2} \mathrm{e}^{j 4 \phi_{c}} \\
C_{42}=M_{42}-\left|M_{20}\right|^{2}=-p^{2} \\
C_{60}=M_{60}-M_{40} M_{20}+3 M_{60}^{3}=4 p^{3} \mathrm{e}^{j 6 \phi_{c}}
\end{gathered}
$$

2) Calculation of each order cumulant of MASK, MFSK, and MPSK signals

Similar to the calculation process of the cumulants of the 2ASK signals, the theoretical values of the cumulants of the MASK, MFSK, and MPSK signals are calculated. 2ASK, 4ASK, 2FSK, 4FSK, 2PSK, 4PSK signal 2nd order, 4th order, 6 th order cumulant calculation results are shown in Table 1 . The cumulant value of each order takes the modulus, and $p$ is the unit value. The modulus values of the second, fourth, and sixthth order cumulants of the digital modulation signal are shown in Table 2.

From the cumulant moduli calculated in Table 2, it can be seen that the difference between the $2 \mathrm{nd}$, 4 th and 6 th order cumulants of $2 \mathrm{ASK}$ and $4 \mathrm{ASK}$ signals is too small to be recognized according to the theoretical value of cumulants.

Table 1. Theoretical value of $2 \mathrm{nd}, 4$ th, 6 th order cumulants of digital modulation signal.

\begin{tabular}{ccccccc}
\hline Method & $C_{20}$ & $C_{21}$ & $C_{40}$ & $C_{41}$ & $C_{42}$ & $C_{60}$ \\
\hline 2 ASK & $p^{2} \mathrm{e}^{j 2 \phi_{c}}$ & $p$ & $-p^{2} \mathrm{e}^{j 4 \phi_{c}}$ & $-p^{2} \mathrm{e}^{j 4 \phi_{c}}$ & $-p^{2}$ & $4 p^{3} \mathrm{e}^{j 4 \phi_{c}}$ \\
4 ASK & $p^{2} \mathrm{e}^{j 2 \phi_{c}}$ & $p$ & $-p^{2} \mathrm{e}^{j 4 \phi_{c}}$ & $-p^{2} \mathrm{e}^{j 4 \phi_{c}}$ & $-p^{2}$ & $4.63 p^{3} \mathrm{e}^{j 4 \phi_{c}}$ \\
2 FSK & 0 & $p$ & 0 & 0 & $-p^{2}$ & 0 \\
4 FSK & 0 & $p$ & 0 & 0 & $-p^{2}$ & 0 \\
2 PSK & $p^{2} \mathrm{e}^{j 2 \phi_{c}}$ & $p$ & $-2 p^{2} \mathrm{e}^{j 4 \phi_{c}}$ & $-2 p^{2} \mathrm{e}^{j 4 \phi_{c}}$ & $-2 p^{2}$ & $16 p^{3} \mathrm{e}^{j 4 \phi_{c}}$ \\
4 PSK & 0 & $p$ & $p^{2} \mathrm{e}^{j 4 \phi_{c}}$ & 0 & $-p^{2}$ & 0
\end{tabular}

Table 2. Cumulative modulus of 2nd, 4 th and 6 th order of digital modulation signal.

\begin{tabular}{ccccccc}
\hline Method & $\left|C_{20}\right|$ & $\left|C_{21}\right|$ & $\left|C_{40}\right|$ & $\left|C_{41}\right|$ & $\left|C_{42}\right|$ & $\left|C_{60}\right|$ \\
\hline 2ASK & 1 & 1 & 1 & 1 & 1 & 4 \\
4 ASK & 1 & 1 & 1 & 1 & 1 & 4.63 \\
2FSK & 0 & 1 & 0 & 0 & 1 & 0 \\
4FSK & 0 & 1 & 0 & 0 & 1 & 0 \\
2PSK & 1 & 1 & 2 & 2 & 2 & 16 \\
4PSK & 0 & 1 & 1 & 0 & 1 & 0 \\
\hline
\end{tabular}


So the mean values of 2ASK signal and 4ASK signal are calculated by Formula (2), and then the 2ASK signal and 4ASK signal are centralised by means. The accumulated modulus of 2ASK signal and 4ASK signal after centralised processing are calculated as shown in Table 3. The 6th-order cumulants of 2ASK signal and 4ASK signal are quite different, so 2ASK signal and 4ASK signal can be identified by 6th-order cumulants. Table 2 in the 2FSK, 4FSK signal of the cumulant values are the same, the cumulant value cannot be used to identify the two signals. Therefore, the frequency difference exponential factor of FSK signal is multiplied by (2) [7] [8] [9] [10] [11], and the higher-order cumulant modulus of FSK signal is calculated as shown in Table 3.

\section{Realization and Simulation of Digital Modulation Signal Recognition Method}

\subsection{Modulation Signal Recognition Process Analysis}

The idea of recognition method: using the calculated higher-order cumulant modulus to set up the recognition characteristic parameter, through the recognition characteristic parameter and the set decision threshold contrast to complete the recognition of digital signal modulation way.

Set $\left|C_{42}\right|$ as the feature parameter, from the moduli of $\left|C_{42}\right|$ in Table 2, $\left|C_{42}\right|=2$ of 2PSK signal, $\left|C_{42}\right|=1$ of the other five signals, $\left|C_{42}\right|$ value can be used to recognize 2PSK signal. $f_{X} 1=\left|C_{40}\right| /\left|C_{42}\right|$, calculate $f_{x} 1$ from Table $2, f_{X} 1=0$ for $2 F S K, 4 F S K, f_{X} 1=0$ for $2 A S K, 4 A S K, 4 P S K$ signals. The value of $f_{X} 1$ can identify the signal as $2 A S K, 4 A S K, 4 P S K$, or the signal as $2 F S K, 4 F S K . f_{x} 2=\left|C_{41}\right| /\left|C_{42}\right|$, calculate $f_{x} 2$ from Table $2, f_{x} 2=0$ for $4 P S K$ signal, $f_{x} 2=1$ for $2 A S K$, $4 A S K$ signal. The value of $f_{x} 2$ can identify $4 P S K$, and the other is $2 A S K, 4 A S K$ signal. $f_{x} 3=$ $\left|C_{40}\right| /\left|C_{42}\right|$, the $2 F S K$ signal $f_{x} 3=0.304$ and $4 F S K$ signal $f_{x} 3=0.01$ are calculated from the values in Table 3 . The $2 F S K$ signal and $4 F S K$ signal are recognized by the value of $f_{X} 3$. Set $\left|C_{60}\right|$ as the characteristic parameter, from the modulus of $\left|C_{60}\right|$ in Table 3, $2 A S K\left|C_{60}\right|=2,4 \mathrm{ASK}\left|C_{60}\right|=0.38$, the $2 \mathrm{ASK}$ signal and $4 \mathrm{ASK}$ signal can be distinguished by the value of $\left|C_{60}\right|$. List Table 4 of calculated values for identifying characteristic parameters $\left|C_{42}\right|, f_{x} 1, f_{x} 2, f_{x} 3,\left|C_{60}\right|$.

\subsection{Modulation Signal Recognition Realization Process}

According to the idea of the recognition method in 3.1, the decision recognition

Table 3. Modulus value of digital modulation signal after processing of $2 \mathrm{nd}$, 4 th, 6 th cumulant.

\begin{tabular}{ccccccc}
\hline Method & $\left|C_{20}\right|$ & $\left|C_{21}\right|$ & $\left|C_{40}\right|$ & $\left|C_{41}\right|$ & $\left|C_{42}\right|$ & $\left|C_{60}\right|$ \\
\hline 2ASK & 0.5 & & 0.5 & & & 2 \\
4ASK & 0.375 & & 0.17 & & 0.38 \\
2FSK & 0.5 & 1 & 0.31 & 1 & 1.02 & \\
4FSK & 0.25 & 1 & 0.012 & 0.5 & 1.08 & \\
\hline
\end{tabular}


flow is as follows: first, the radio digital modulation signal to be recognized at the receiving end is mixed into baseband signal; Thirdly, the characteristic parameters $\left|C_{42}\right|, f_{x} 1, f_{x} 2, f_{X} 3,\left|C_{60}\right|$ are set and their values are calculated, and the values of decision threshold th1, th2, th 3 , th 4 , th 5 are set, to run a decision recognition.

The decision recognition process is implemented as follows:

1) According to the value of $\left|C_{42}\right|$, the signals to be identified are divided into two categories: when $\left|C_{42}\right|>t h 1$, the signals to be identified are 2PSK signals; $\left|C_{42}\right|<t h 1$, the signals to be identified are 2ASK, 4ASK, 2FSK, 4FSK and 4PSK signals.

2) Calculate the identification characteristic parameter $f_{x} 1$, if $f_{x} 1<t h 2$, determine the digital modulation signal to be identified as 2FSK, 4FSK signals; if $f_{x} 1>t h 2$, determine the digital modulation signal to be identified as 2ASK, 4ASK, 4PSK signals.

3) Calculate the identification characteristic parameter $f_{x} 2$, if $f_{x} 2<t h 3$, determine that the digital modulation signal to be identified is 4PSK signal, if $f_{x} 2>$ th3, then determine that the digital modulation signal to be identified is 2ASK, 4ASK signal.

4) Calculate the identification characteristic parameter $f_{x} 3$, if $f_{x} 3<t h 4$, determine that the digital modulation signal to be identified is a 4FSK signal, if $f_{x} 3>$ th4 , determine that the digital modulation signal to be identified is a $2 \mathrm{FSK}$ signal.

5) Calculate the identification characteristic parameter $\left|C_{60}\right|$, if $\left|C_{60}\right|<t h 5$, then determine that the digital modulation signal to be identified is a 4ASK signal, if $\left|C_{60}\right|>t h 5$, then determine that the digital modulation signal to be identified is a 2ASK signal.

The block diagram of the process of identifying digital modulation signals is shown in Figure 1.

\subsection{Simulation Realization of Modulation Signal Recognition Process}

Use MATLAB programming file to realize the whole process of wireless digital modulation signal recognition and simulation.

1) Simulation parameter setting: software MATLAB2016b, signal carrier frequency $f_{\mathcal{C}}=10 \mathrm{MHz}$, sampling frequency $f_{s}=60 \mathrm{MHz}$, symbol rate is $2.5 \mathrm{MBaud}$, Gaussian white noise. Each simulation sends 1000 symbols, and the decision threshold is set: th $1=1.5$, th $2=0.5$ th $3=0.51$, th $4=0.15$, th $5=1.19$. The SNR at the receiving end: $-2 \mathrm{~dB}-16 \mathrm{~dB}$, repeat the simulation experiment 50 times.

2) The process of determining characteristic parameters and threshold values of recognition: carry on the simulation to the digital modulation signal 2ASK, 4ASK, 2FSK, 4FSK, 2PSK, 4PSK recognition characteristic parameter $\left|C_{42}\right|, f_{x} 1$, $f_{X} 2, f_{X} 3,\left|C_{60}\right|$, the simulation results show that the theoretical values of $\left|C_{42}\right|, f_{X} 1$, $f_{X} 2, f_{X} 3,\left|C_{60}\right|$ can be used as the recognition parameters of digital modulation signals. The simulation results of the $\left|C_{42}\right|$ (Figure 2) show that 2PSK signals 


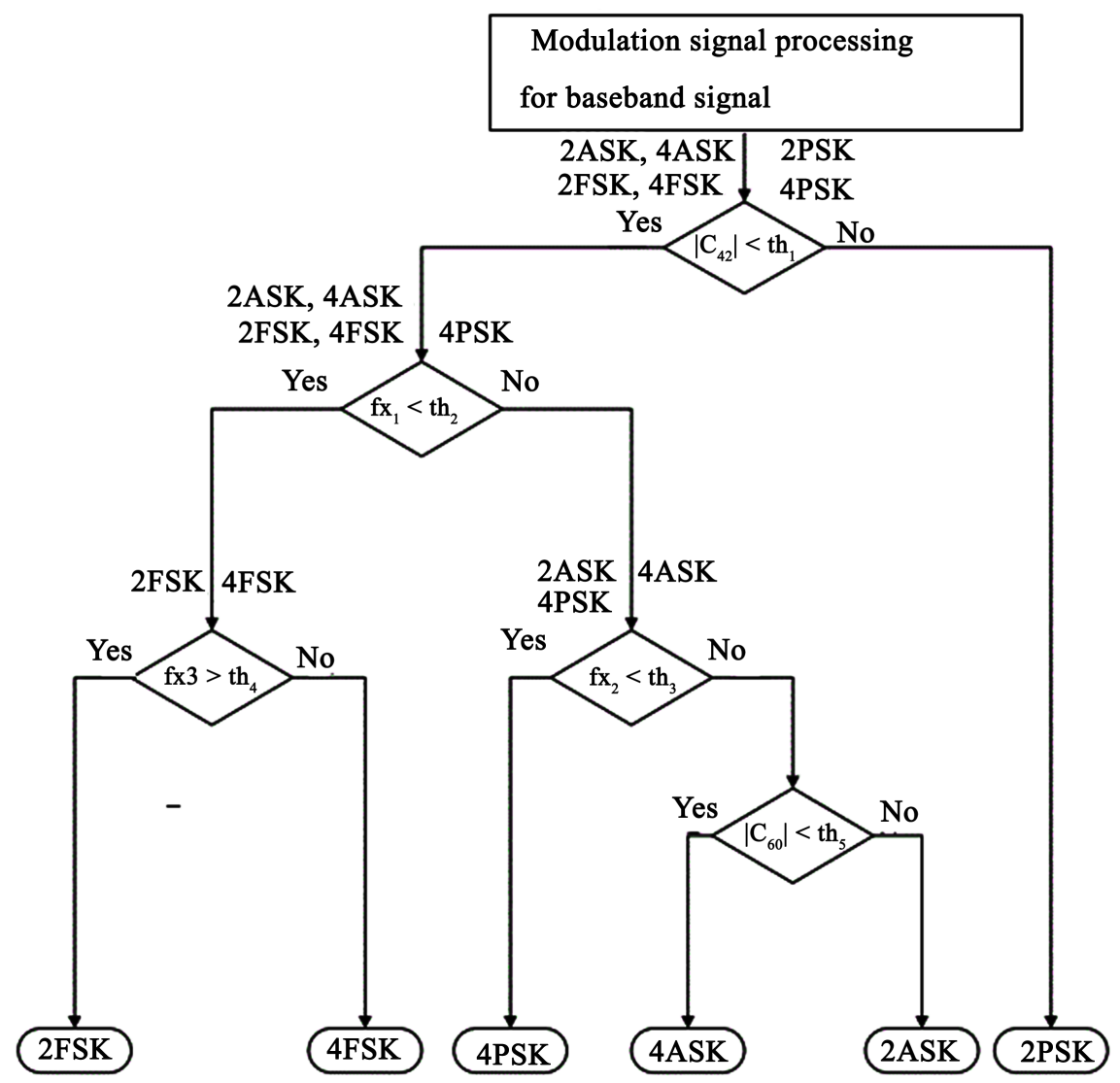

Figure 1. Block diagram of the process of identifying digital modulation signals.

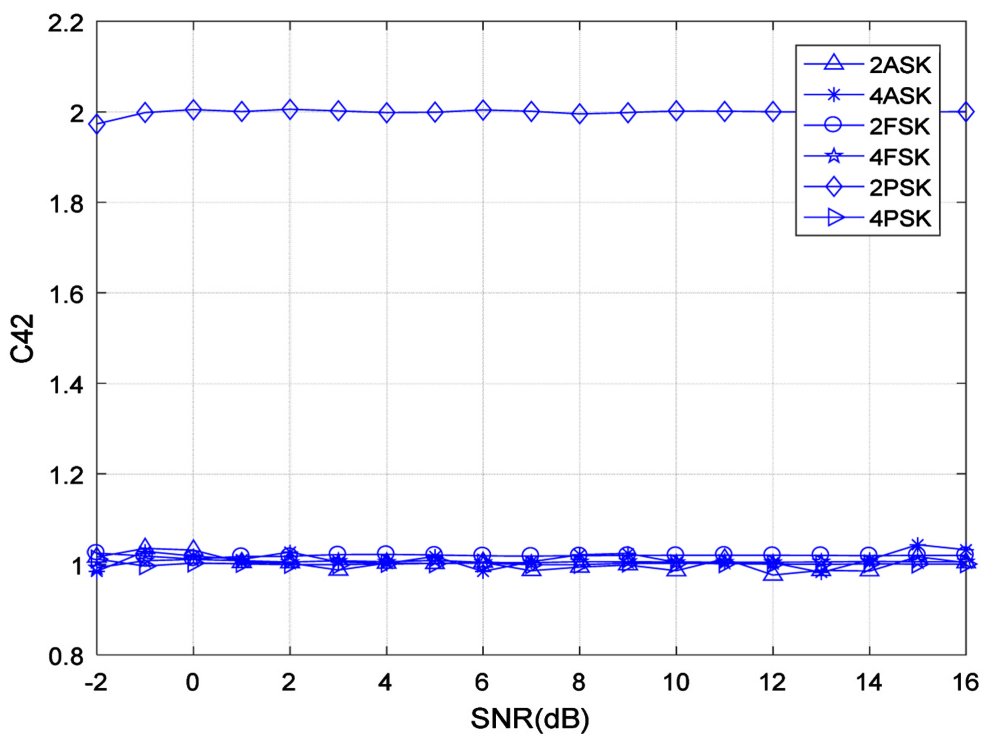

Figure 2. Simulation of $\left|C_{42}\right|$ value.

$\left|C_{42}\right|=2$, 2ASK, 4ASK, 4PSK, 2FSK, 4FSK signals $\left|C_{42}\right|=1,\left|C_{42}\right|$ is consistent with the theoretical value in Table 4. $\left|C_{42}\right|$ can divide the digital modulation signals to be recognized into two kinds, and take the mean value of $t h 1=1.5\left(\left|C_{42}\right|\right)$. $f_{X} 1$ simulation results (Figure 3 ) show that $f_{x} 1 \approx 0$ of $2 \mathrm{FSK}$ and $4 \mathrm{FSK}$ signals, 


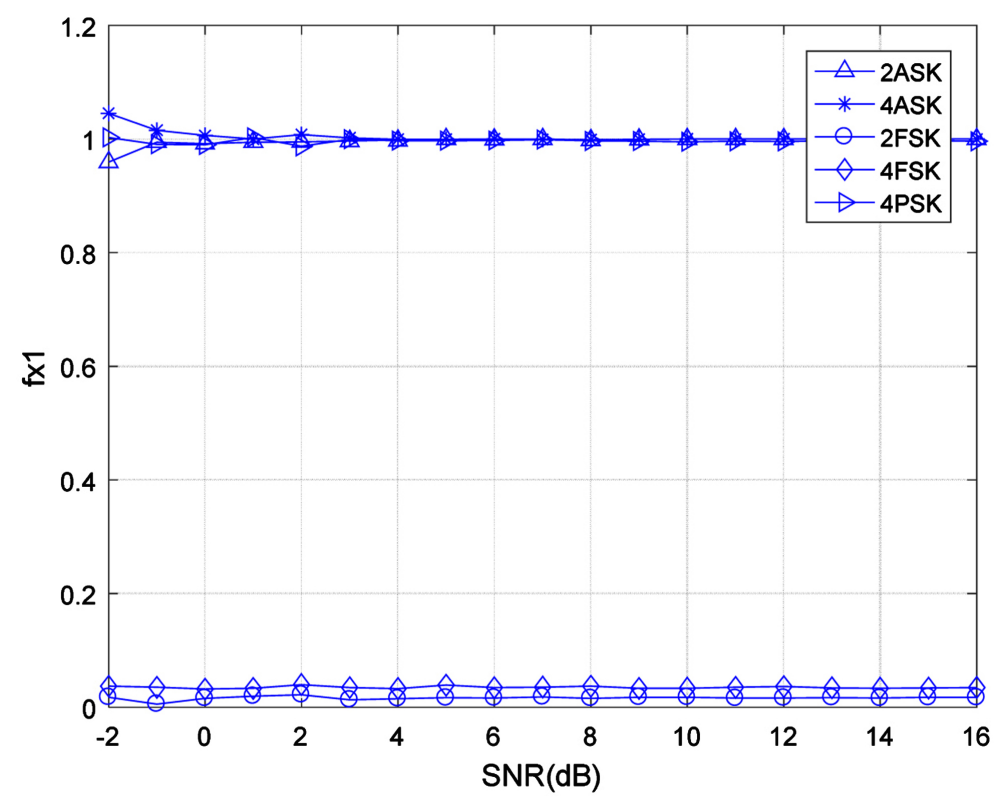

Figure 3. Simulation of $f_{x} 1$ value.

Table 4. Identify the value of the feature parameter.

\begin{tabular}{cccccc}
\hline Method & $\left|C_{42}\right|$ & $f_{x} 1$ & $f_{x 2}$ & $f_{x 3}$ & $\left|C_{60}\right|$ \\
\hline 2 ASK & 1 & 1 & 1 & & 2 \\
4 ASK & 1 & 1 & 1 & & 0.38 \\
FFSK & 1 & 0 & & 0.304 & \\
4 FSK & 1 & 0 & & 0.01 & \\
2PSK & 2 & 1 & 0 & & \\
4 PSK & 1 & 1 & & \\
\hline
\end{tabular}

while $f_{x} 1 \approx 1$ of $2 \mathrm{ASK}, 4 \mathrm{ASK}$ and $4 \mathrm{PSK}$ signals, the simulation values of $f_{x} 1$ are consistent with the theoretical values of Table 4 . $f_{x} 1$ can be used to classify and identify the digital modulation signals to be recognized, and th2 $=0.5$. $f_{x} 2$ as shown in Figure 4, 4PSK signal characteristic parameter $f_{x} 2 \approx 0$, 2ASK, 4ASK signal characteristic parameter $f_{x} 2=1, f_{x} 2$ simulation results are consistent with the theoretical values of $f_{x} 2$ in Table $4 . f_{x} 2$ is used to classify the digital modulation signals to be identified into two kinds: 2ASK, 4ASK and 4PSK, th3 $=0.51$. $f_{X} 3$ simulation results Figure 5 shows that the 2FSK signal characteristic parameter $f_{X} 3 \approx 0.30,4 \mathrm{FSK}$ signal characteristic parameter $f_{X} 3 \approx 0.01$. The theoretical value of $f_{x} 3$ in Table 4 is basically consistent with the simulation results. Selecting th $4=0.15, f_{X} 3$ can realize the classification of 2 FSK signal and 4FSK signal. $\left|C_{60}\right|$ simulation results are shown in Figure 6. Figure 6 shows that 2ASK signal $\left|C_{60}\right|=1.99$, 4ASK signal $\left|C_{60}\right|=0.38$. $\left|C_{60}\right|$ simulation results agree with the theoretical value in Table 4 , take $t h 5=1.19$. The simulation verifies the correctness of the set decision threshold and the set recognition feature parameters. 


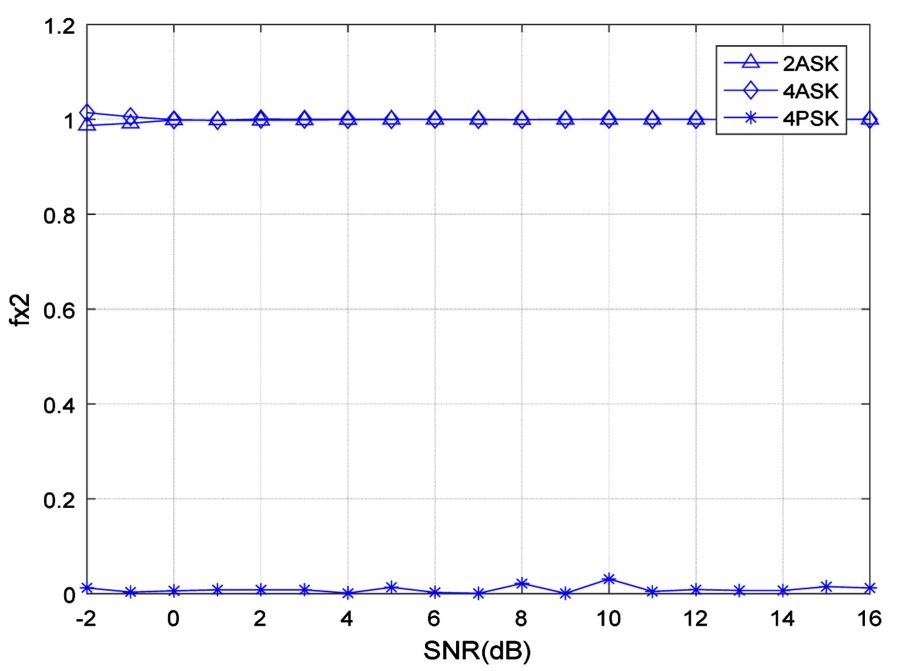

Figure 4. Simulation of $f_{x} 2$ value.

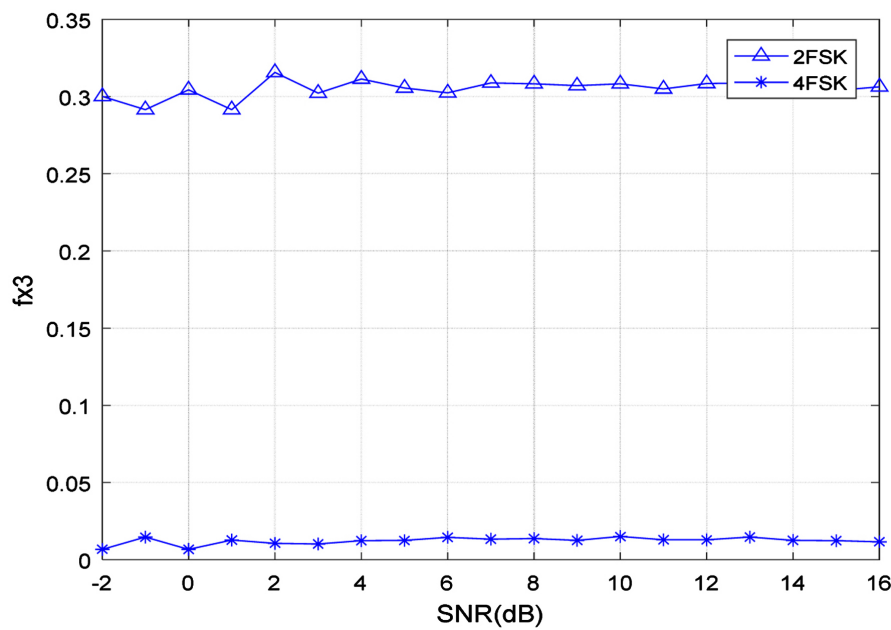

Figure 5. Simulation of $f_{x} 3$ value.

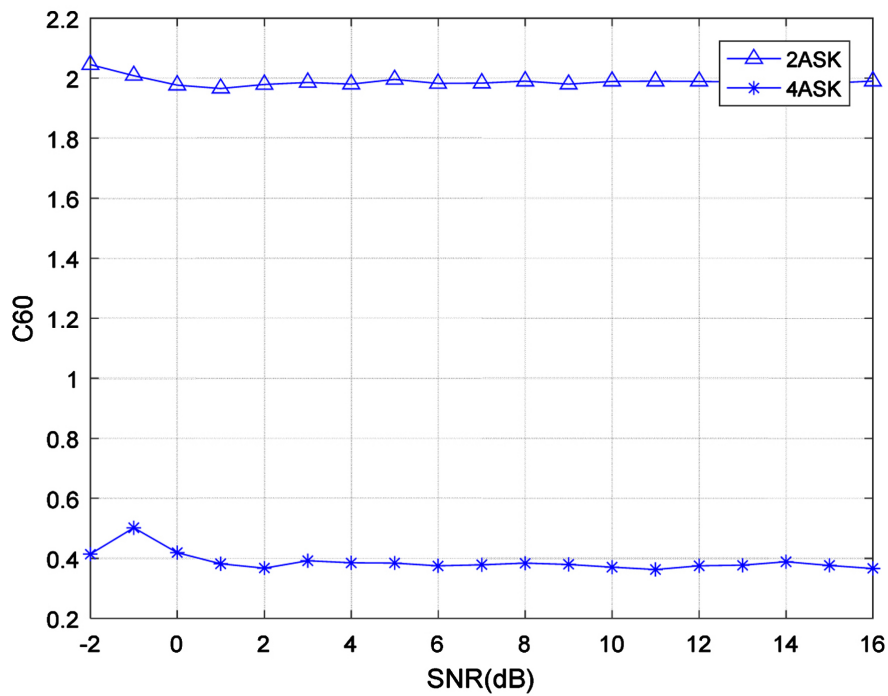

Figure 6. Simulation of $\left|C_{60}\right|$ value. 


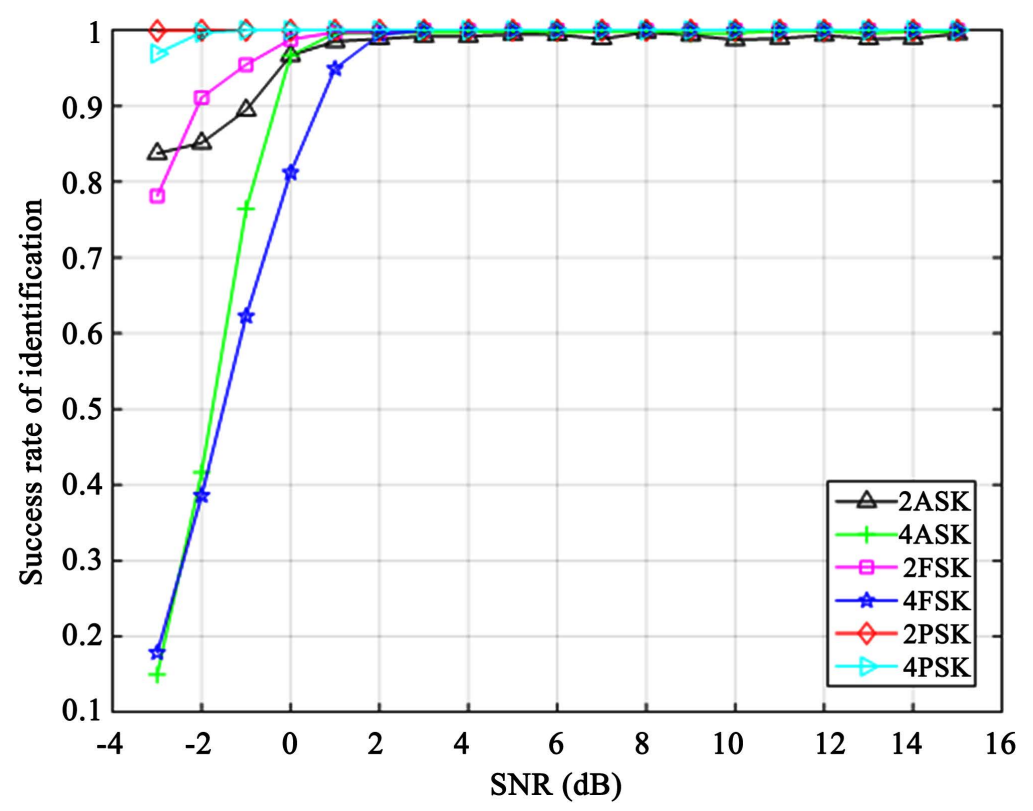

Figure 7. Recognition rate of six digital modulation signals.

3) Digital modulation signal recognition simulation: according to the recognition flow chart 1 programming, the recognition rate of the results shown in Figure 7. Figure 7 shows that when SNR is greater than 0 , the recognition success rate of $4 \mathrm{ASK}$ signal is over $80 \%$, while that of $2 \mathrm{ASK}, 2 \mathrm{PSK}, 4 \mathrm{PSK}, 2 \mathrm{FSK}, 4 \mathrm{FSK}$ signal are all over $85 \%$. When SNR is greater than $2 \mathrm{~dB}$, the recognition rate of the six kinds of signals is $99 \%$, so the recognition method of this kind of digital modulation signals is still good in the case of poor signal-to-noise ratio. Therefore, the method of higher-order cumulant has a good anti-noise ability to the digital modulation signal recognition method, and has a higher recognition rate even under the lower SNR. At the same time, the simulation results show that the simulation recognition process of this method is realized by calculating five characteristic parameter values, which has the characteristics of less calculation data, simple judgment process and fast recognition.

\section{Conclusion}

By using 2ASK, 4ASK, 2FSK, 4FSK, 2PSK, 4PSK signals, the feasibility of using high-order cumulants of modulation signals to construct characteristic parameters and realize the recognition of digital modulation signals is verified by simulation. At the same time, the simulation results show that: a wireless digital modulation signal recognition system is designed based on the analysis method, which realizes the recognition function and has a high correct recognition rate. Especially in the case of poor signal-to-noise ratio at the receiving end, it also has a good recognition effect. Although six kinds of signals are taken as examples to verify the simulation, the principle of the recognition method and the recognition system can be extended to recognize more modulated signals, such as MASK, MFSK, MPSK and MQAM. As a follow-up task, the recognition analysis 
simulation of other modulation modes is carried out. At the same time, it should be noted that the wireless digital signal cumulant discussed in this paper is analyzed by one-way signal, and the wireless digital modulation signal may appear one-way or multi-way at the same time at the receiver. It is necessary to distinguish the mixed digital modulation signals of the receiver into a single one-way and transform them into baseband signals. In a word, wireless digital modulation signal recognition is an important technology, and we need to conduct in-depth study of this theory and practical applications.

\section{Conflicts of Interest}

The authors declare no conflicts of interest regarding the publication of this paper.

\section{References}

[1] Jyoti, P.G. and Kumar, B.P. (2016) Parameter Estimation for Blind Classification of Digital Modulations. IET Signal Processing, 10, 758-769. https://doi.org/10.1049/iet-spr.2015.0373

[2] Zhao, Y., Xu, Y.T., Jiang, H., et al. (2015) Recognition of Digital Modulation Signals Based on High-Order Cumulants. 2015 International Conference on Wireless Communications \& Signal Processing ( WCSP), Nanjing, 15-17 October 2015, 1-5. https://doi.org/10.1109/WCSP.2015.7341279

[3] Simic, M., Stankovic, M. and Orlic, V.D. (2021) Automatic Modulation Classification of Real Signals in AWGN Channel Based on Sixth-Order Cumulants. Radio Engineering, 30, 204-214. https://doi.org/10.13164/re.2021.0204

[4] Abdelmutalab, A., Assaleh, K. and El-Tarhuni, M. (2016) Automatic Modulation Classification Based on High Order Cumulants and Hierarchical Polynomial Classifiers. Physical Communication, 21, 10-18. https://doi.org/10.1016/j.phycom.2016.08.001

[5] Zhu, W., et al. (2021) Research on Improved Time Difference Estimation Algorithm Based on Fourth-Order Cumulant in UHF PD Location. Journal of Physics: Conference Series, 1983, Article ID: 012062. https://doi.org/10.1088/1742-6596/1983/1/012062

[6] Daoud, S. and Ghrayeb, A. (2016) Using Resampling to Combat Doppler Scaling in UWA Channels with Single-Carrier Modulation and Frequency-Domain Equalization. IEEE Transactions on Vehicular Technology, 65, 1261-1270. https://doi.org/10.1109/TVT.2015.2409560

[7] Jammalamadaka, S.R., Taufer, E. and Terdik, G.H. (2021) Asymptotic Theory for Statistics Based on Cumulant Vectors with Applications. Scandinavian Journal of Statistics, 48, 708-728. https://doi.org/10.1111/sjos.12521

[8] Long, H. and Zhao, J. (2014) Identification of Multiple Inputs Single Output Errors-in-Variables System Using Cumulant. Journal of Systems Engineering and Electronics, 25, 921-933. https://doi.org/10.1109/JSEE.2014.00106

[9] Wang, A. and Liu, P. (2020) Digital Signal Modulation Recognition Method Based on High-Order Cumulants and Wavelet Transform. Conference Proceeding of 2020 3 rd International Conference on Algorithms, Computing and Artificial Intelligence (ACAI2020), Sanya, December 2020, 554-559.

https://doi.org/10.1145/3446132.3446423 
[10] Yang, Y., Jiang, S. and Zhou, Z. (2019) Research on Signal Models and Recognition Method for Digital Modulation. IEEE 7 th International Conference on Computer Science and Network Technology (ICCSNT 2019), Dalian, 19-20 October 2019, 519-523.

[11] Zhou, X., Wu, Y. and Yang, B. (2010) Signal Classification Method Based on Support Vector Machine and High-Order Cumulants. Wireless Sensor Network, 2, 48-52. https://doi.org/10.4236/wsn.2010.21007 